\title{
Interaction between Shock Wave and Boundary Layer in Nonequilibrium Hypersonic Rarefied Flow* (5th Report, Nonequilibrium of Rotational Temperature in Flow over Flat Plate)
}

\author{
Nobuyuki TSUBOI $^{* *}$ and Yoichiro MATSUMOTO***
}

\begin{abstract}
An experimental study of the interaction between a shock wave and a boundary layer over a flat plate with a sharp leading edge in hypersonic rarefied gas flow is presented. Experiments in a low-density wind tunnel using an electron beam probe were conducted at the Shock Wave Laboratory, RWTH Aachen, Germany. Rotational temperatures for stagnation temperatures of $T_{0}=670 \sim 1000 \mathrm{~K}$ and $K n=0.024 \sim 0.028$ based on a reference length of $0.05 \mathrm{~m}$ were calculated using Muntz's method and Robben and Talbot's method. The domain of quasi two-dimensional flow over the plate was determined from three-dimensional rotational temperature measurements. Nonequilibrium between translational and rotational temperatures was observed near the leading edge, and the experimental rotational relaxation length explains the rotational collision number of $2 \sim 4$.
\end{abstract}

Key Words: Rarefied Gas Flow, Hypersonic Flow, Nonequilibrium, Rotational Temperature

\section{Introduction}

One of the important phenomena in aerodynamics is the determining the mechanism underlying an interaction between a shock wave and a boundary layer at the reentry in the atmosphere, although the development of aerospace technology has recently generated a strong demand for research associated with rarefied gas dynamics. In the highaltitude and low-density environment, flow separation is restrained to affect the aerodynamic characteristic parameters such as the pitching moment. Therefore, the fundamental physical phenomena such as the shock wave and boundary layer interaction in the rarefied regime have to be investigated.

There are two typical phenomena in the shock wave and boundary layer interaction; one is due to the shock

* Received 24th April, 2006 (No. 04-0378). Japanese Original: Trans. Jpn. Soc. Mech. Eng., Vol.71, No.702, B (2005), pp.420-427 (Received 25th June, 2004)

** Space Transportation Engineering Department, Institute of Space and Astronautical Science, Japan Aerospace Exploration Agency, 3-1-1 Yoshinodai, Sagamihara, Kanagawa 229-8510, Japan

*** Department of Mechanical Engineering, The University of Tokyo, 7-3-1 Hongo, Bunkyo-ku, Tokyo 113-8656, Japan wave incident on the boundary layer, and the other is a viscous interaction in the vicinity of a flat plate with a sharp leading edge. The former phenomenon is the separation of a boundary layer due to a strong shock wave incident on the boundary layer and the latter one is an interaction between a free stream and a rapidly developing boundary layer. The present paper focuses on the latter interaction. The flow structure in the region dominant such the interaction and the nonequilibrium phenomenon between translational and rotational temperatures are unclear.

In the first, second and third reports ${ }^{(1)-(3)}$, we revealed the nonequilibrium state between translational and rotational temperatures near the wall of the sharp flat plate using the direct simulation Monte Carlo (DSMC) $\operatorname{method}^{(6),(7)}$ with the Dynamic Molecular Collision (DMC) model ${ }^{(4)}$ and the Multi-Stage (MS) model ${ }^{(5)}$. The multiscale method proposed by the authors is shown to be able to give reasonably good simulation results.

However, the calculated results, especially nonequilibrium rotational temperatures, have not been compared with the experimental results. In the 1970s, rotational temperature was measured using the electron beam fluorescence method (EBFM) by some researchers ${ }^{(8),(9)}$, however, these measurements have mainly been conducted in a free-expansion jet or a normal shock wave. Therefore, there is little experimental data measured in the interaction 
between a shock wave and a boundary layer. Even though a freestream flow has to be generated in order to study such an interaction using a nozzle, Reynolds number becomes low and the thickness of the boundary layer becomes large in such a low-density region. In the fourth report ${ }^{(10)}$, we designed a conical nozzle for the low-density wind tunnel and obtained a core diameter of $3 \mathrm{~cm}$ and an exit Mach number of 4.8. DSMC simulation was also carried out to agree well with the experimental results ${ }^{(11)}$. In this report, we present the nonequilibrium rotational temperature distributions over the flat plate measured using the EBFM.

\section{Nomenclature}
$E$ : Energy
$I:$ Spectrum intensity
$k$ : Boltzmann constant
$K$ : Rotational level

$K \max$ : Maximum rotational level

$K n:$ Knudsen number

$L:$ Reference length

$L E$ : Leading edge angle

$M$ : Mach number

$p$ : Pressure

$P$ : Transition probability

$R e$ : Reynolds number

$T$ : Temperature

$X, Y, Z$ : Location in $x, y, z$ coordinates

$\rho:$ Density

\section{Experimental Method}

\subsection{Experimental setup}

Experiments were conducted in a low-density wind tunnel constructed by late Prof. Beylich ${ }^{(12)}$ at the SWL (Shock Wave Laboratory), RWTH Aachen, Germany. Free jet experiments were conducted using an electron beam in a low-density wind tunnel ${ }^{(12)}$ in the 1970 s and the present study involves rotational temperatures measured using these apparatuses.

The wind tunnel consists of two crossed cylinders made of steel with diameters of $1.8 \mathrm{~m}$ and $2.4 \mathrm{~m}$ and lengths of $8.1 \mathrm{~m}$ and $4 \mathrm{~m}$, respectively. The net volume of the vacuum tank is $28 \mathrm{~m}^{3}$. The electron beam gun, heating chamber, three-dimensional transverse unit, and optical lenses are mounted inside the wind tunnel. The spectrometer, mass flow control facility, and other units are outside the tunnel. Water cooling pipes coming from outside the tunnel are used to cool the electron beam gun, among others. Two rotary pumps, one root pump, and one oil vapor booster pump are connected to the wind tunnel and allow a steady-state operation. These pumps are also cooled by a closed water system connected to a heat exchanger outside the laboratory. The final pressure with all the pumps running is about $0.1 \mu$ bar $(0.01 \mathrm{~Pa})$ without gas flowing in the tunnel.

Figure 1 shows a schematic diagram of the wind tunnel and of the measurement facilities. The electron beam gun is fixed to the tank top of the test section. This beam gun has a maximum acceleration voltage of $40 \mathrm{kV}$ and a maximum anode current of $10 \mathrm{~mA}$. This beam is sent through an orifice of about $1 \mathrm{~mm}$ diameter into the chamber and ends in a Faraday cage.

The stagnation chamber and flat plate are connected to the transverse unit. The stagnation temperature and stagnation pressure are measured in the stagnation chamber. Both the static pressure at the nozzle exit, and the wall temperature at the flat plate are measured. The measured data are converted into electronic signals by an AD converter, and stored in the PC outside the wind tunnel. The Pitot pressures at the nozzle exit are measured without the flat plate. The light signal of the fluorescence created by the electron beam gun passes through the lenses and is recorded by the spectrometer and photomultiplier. Optical

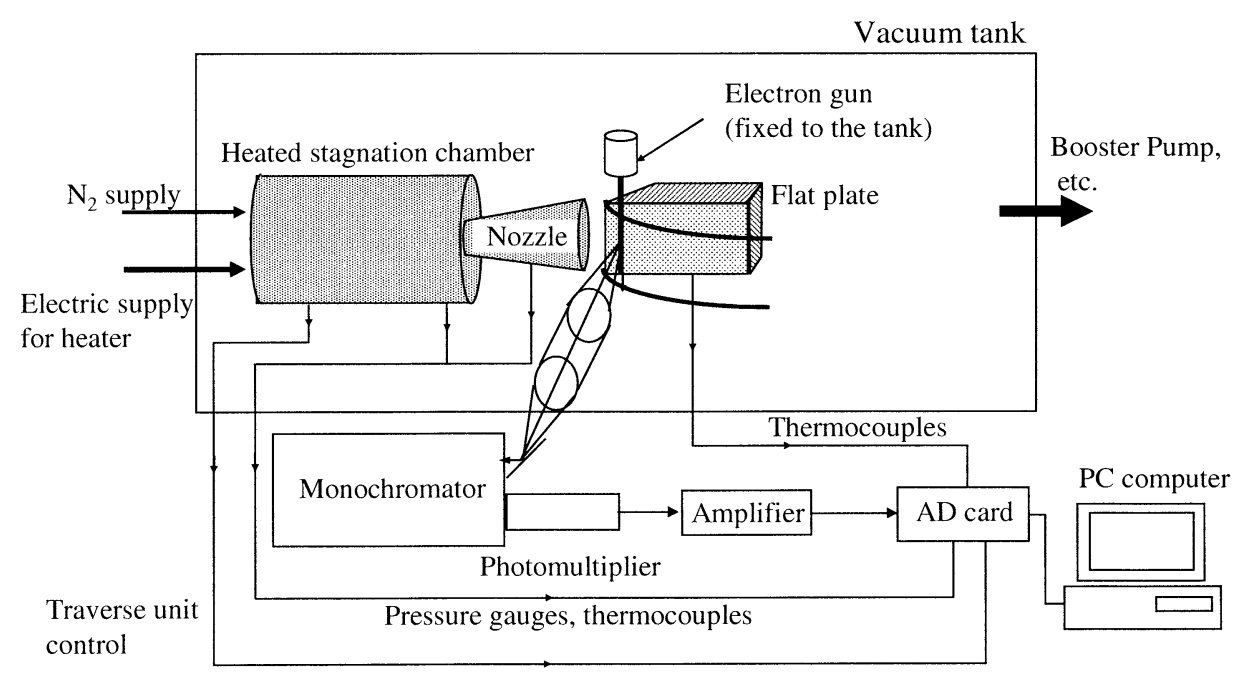

Fig. 1 Setup of measurement system 
data are also stored in the PC. Three stepping motors connected to the transverse unit inside the tank can change the $x, y, z$-position of the stagnation chamber. These motors are controlled by the same PC. The experimental apparatus is described in detail in Refs. (12) and (13).

\subsection{Pressure measurement}

Pressures in the tank and at the nozzle exit vary from $0.01 \mathrm{~Pa}$ to $300 \mathrm{~Pa}$, and are measured using differential pressure gauges (MSK Company). As the pressure in the stagnation chamber and the Pitot pressure vary over a wide range of values, from $10 \mathrm{~Pa}$ to $2000 \mathrm{~Pa}$, two absolutepressure gauges (MKS Company) are used. To estimate the thickness of the boundary layer at the nozzle exit, the Pitot pressure was measured using a Pitot pressure probe with an inner diameter of $3.85 \mathrm{~mm}$. The local Mach number and the static temperature at the nozzle exit are then calculated from the Pitot pressure data at the nozzle exit and from the stagnation pressure. The Mach number and the temperature at the nozzle exit are also calculated from the measured static pressure at the nozzle exit. In the present experiment, a static pressure port with the diameter of $5 \mathrm{~mm}$ is used at a location situated $6 \mathrm{~mm}$ upstream from the nozzle exit.

\subsection{Rotational temperature measurement}

Rotational temperature was measured using the electron beam fluorescence method (EBFM). This method, which was originally proposed and developed by Muntz ${ }^{(8)}$, has been widely used in the past to measure the rotational temperature in a rarefied gas flow. The rotational temperature measurement by the EBFM technique has mainly been conducted in a free expansion jet for the stagnation temperature of room temperature ${ }^{(8),(14)-(17)}$. When a beam of electrons is passed through nitrogen gas, a nitrogen molecule, $N_{2} X^{1} \Sigma_{g}^{+}$, with an initial electronic state $E_{1}^{\prime \prime}$, is excited to an ionized nitrogen molecule, $N_{2} B^{2} \Sigma_{u}^{+}$, with a state $E^{\prime}$ by an inelastic collision with an electron. Emission of a photon from $E^{\prime}$ brings the molecule back to an ionized nitrogen molecule, $N_{2} X^{2} \Sigma_{q}^{+}$, with a ground state $E_{2}^{\prime \prime}$. This fluorescence is called the "first negative system". In order to calculate the rotational temperature, the fluorescence in the $\mathrm{R}$ branch of the band spectrum at $391.4 \mathrm{~nm}$ $((0,0)$ transition) was measured using the monochromator. The measured spectrums are the $\mathrm{P}$ branch and $\mathrm{R}$ branch, which are generated due to the transition of the rotational levels $\Delta K= \pm 1$ in the "first negative system". Therefore, $\Delta K=+1$ is the $\mathrm{R}$ branch and $\Delta K=-1$ is the $\mathrm{P}$ branch, respectively. Both branches can be measured to calculate the rotational temperature. The wavelength between each level in the $\mathrm{P}$ branch is smaller than that in the case of the $\mathrm{R}$ branch; therefore, the $\mathrm{R}$ branch is valid for us to resolve the wavelength using the monochromator. The rotational temperatures are calculated from the measured spectrums in the R branch and can be calculated by two methods: the Muntz model ${ }^{(8)}$ and Robben and Talbot (RT) model ${ }^{(18)}$. The Muntz model uses the relation between intensity distribution in the rotational structure of the fluorescence and the initial population distribution through an appropriate model of the excitation-emission process. In the Muntz model, the excitation process uses the dipole approximation. In an equilibrium state, the Muntz model is able to define a unique rotational temperature because all rotational levels are fitted by a straight line. However, in a nonequilibrium state, a unique rotational temperature cannot be defined because nonequilibrium rotational distributions create an overpopulation of the higher rotational levels. The RT model is based on the same concept as the Muntz model. It uses the merging of two rotational distribution functions at temperatures calculated from the lower and higher rotational levels. It defines an averaged rotational temperature $T_{r o t}$ as $T_{r o t}=E_{r o t} / \rho$, where $E_{r o t}$ is the sum of rotational energies and $\rho$ is the density of molecules. The RT model also uses the dipole approximation in the excitation process.

Figure 2 shows an example of rotational spectra of the (0-0) vibrational band of the $N_{2}^{+}$first negative system for two different slit widths. It is possible to isolate the contribution of each overlapping spectrum to the complete band by assuming each rotational spectrum to be a Gaussian distribution ${ }^{(19)}$. The results are shown as the fitting function in Fig. 2. Using the intensities deduced from fitting the spectra, $\log \left(I_{K} / P_{K}\right)$ vs. $E_{\text {rot }} / k$ can be calculated. The results are shown in Fig. 3. The intensities are not distributed along a straight line because of the strong nonequilibrium flow over the plate. To analyze these data, we calculated two temperatures. The first one is a rotational temperature calculated by fitting the points for $1<K<5$ and $15<K<19$ using the Muntz method. The second one is calculated using the RT method in which $K^{\prime}$ is chosen as Kmax-5. According to Fig. 3, $K^{\prime}$ in Eqs. (8) and (19) in Ref.(18) is set to 15. The five lowest levels $(1<K<5)$ are fitted by the full straight line and the five highest levels $(15<K<19)$ are fitted by the broken straight line. The detailed procedure is mentioned in Ref. (19).

\subsection{Stagnation chamber, nozzle and flat plate}

The stagnation chamber has two heat sources and three heat shields to reduce radiation. It has an effective volume of about $0.32 \mathrm{~m}^{3}$ and is made of a stainless-steel tube with a diameter of $86 \mathrm{~mm}$ and a length of $300 \mathrm{~mm}$. The heating element of the chamber is made of nicrome and the empty space in the chamber is filled with ceramic balls of $5 \mathrm{~mm}$ diameter to enhance heat transfer to the nitrogen gas. The chamber is operated to a maximum temperature of $T_{0}=1000 \mathrm{~K}$.

A convergent-divergent nozzle was designed for the present experiment. The detailed procedure for the design and the results are presented in Ref. (10) and the summary is described as follows. The nozzle exit half angle is 


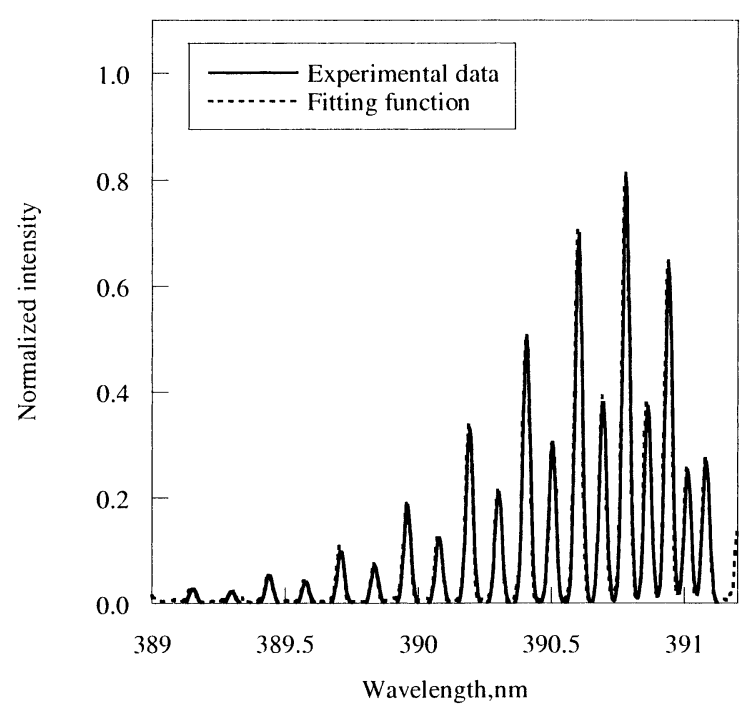

(a) Run 34 with slit width of $80 \mathrm{~nm}$

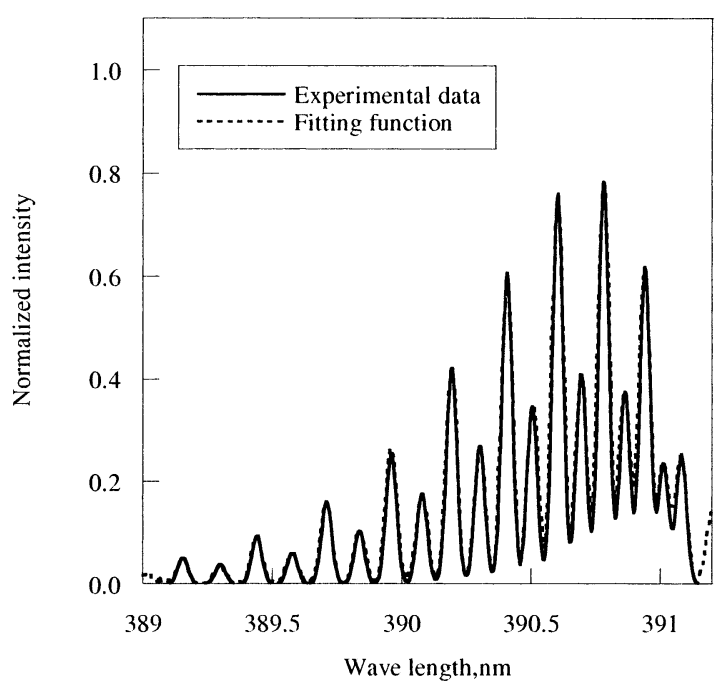

(b) Run 48 with slit width of $120 \mathrm{~nm}$

Fig. 2 Rotational spectra of (0-0) vibrational band of $N_{2}^{+}$first negative system in nitrogen flow at $X=5 \mathrm{~mm}$ and $Y=3 \mathrm{~mm}$ and fitting function

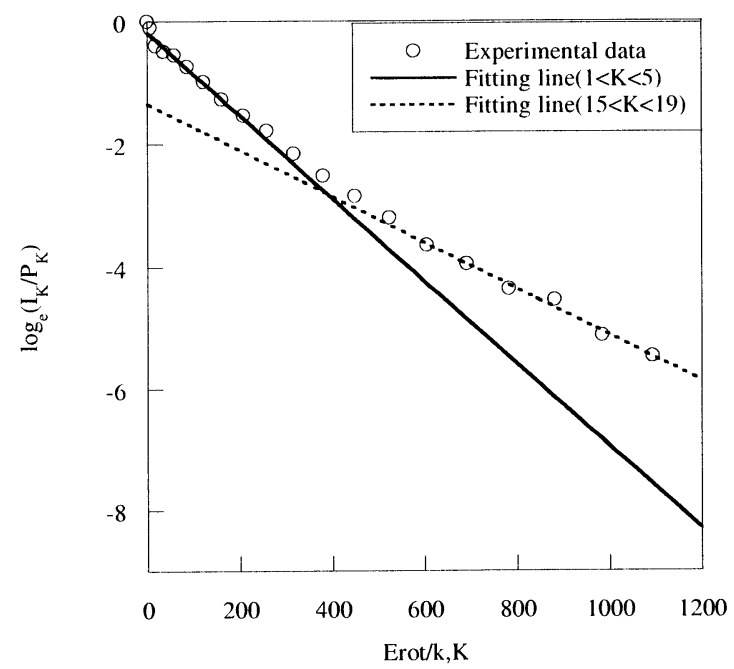

(a) Run 34 with slit width of $80 \mathrm{~nm}$

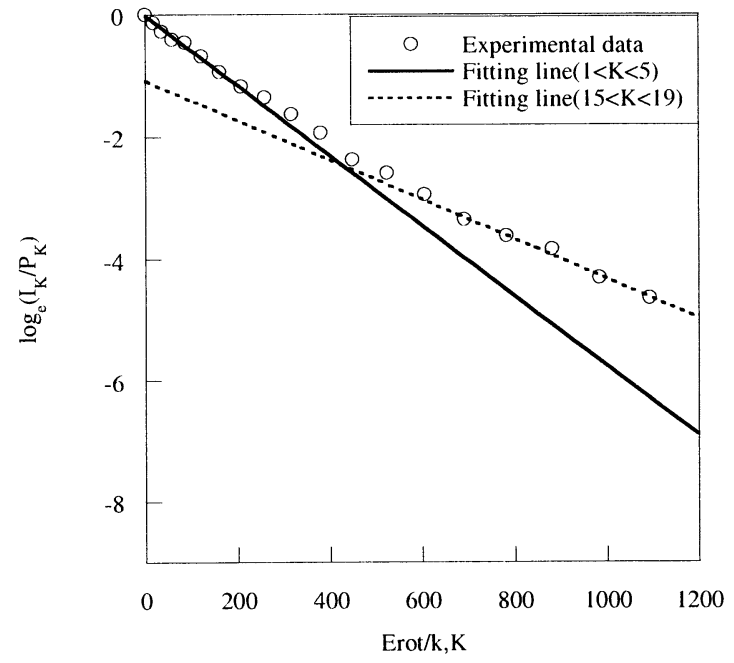

(b) Run 48 with slit width of $120 \mathrm{~nm}$

Fig. 3 Plot of $\log \left(I_{K} / P_{K}\right)$ vs. $E_{\text {rot }} / k$ calculated from Fig. 2

$15 \mathrm{deg}$. and the exit diameter is $10 \mathrm{~cm}$. The nozzle is made of stainless steel. In order to cool the nozzle wall and reduce the thickness of the wall boundary layer, a copper tube with a diameter of $8 \mathrm{~mm}$ for water cooling was wound around the nozzle from the throat to the nozzle exit.

The flat plate was made of copper because the plate should be cooled by water to maintain a constant wall temperature. The leading edge angle is $30 \mathrm{deg}$., the plate thickness is $15 \mathrm{~mm}$, the plate width is $100 \mathrm{~mm}$ and the plate length is $130 \mathrm{~mm}$. The leading edge is sharp. The distance between the nozzle exit and the leading edge of the plate is $15 \mathrm{~mm}$. Thermocouples were fixed in the plate in order to measure the plate wall temperature. Two copper pipes for cooling were soldered to the lower side of the plate.
The flat plate is supported from the rear side by a straight sting. The flat plate, stagnation chamber and nozzle move together whereas the electron beam gun is fixed in the wind tunnel. The sting was mounted from the side of the stagnation chamber. The plate location, height and angle of attack can be varied using the sting support system. The plate surface is parallel to the electron beam as shown in Fig. 1.

\section{Results and Discussions}

Experimental conditions are shown in Table 1. The gas state at the nozzle exit is calculated from the nozzle exit static pressure.

The results of the experimental study are discussed as follows: 
Table 1 Experimental conditions

\begin{tabular}{|c|c|c|c|}
\hline Run number & 26 & 34 & 48 \\
\hline Configuration $^{(* 1)}$ & Nozzle & $\begin{array}{c}\text { Plate } \\
\text { LE30 }\end{array}$ & $\begin{array}{c}\text { Plate } \\
\text { LE30 }\end{array}$ \\
\hline $\begin{array}{c}\text { Nozzle exit } \\
\text { Mach number } M_{e}\end{array}$ & 4.85 & 4.89 & 4.92 \\
\hline $\begin{array}{c}\text { Stagnation temperature } \\
T_{0}\lceil\mathrm{~K}]\end{array}$ & 680 & 670 & 999 \\
\hline $\begin{array}{c}\text { Stagnation pressure } \\
p_{0}\lceil\text { Pa }]\end{array}$ & 965 & 983 & 1271 \\
\hline $\begin{array}{c}\text { Nozzle exit } \\
\text { temperature } T_{e}[\mathrm{~K}]\end{array}$ & 119 & 116 & 171 \\
\hline $\begin{array}{c}\text { Nozzle exit } \\
\text { static pressure } p_{e}[\mathrm{~Pa}]\end{array}$ & 2.18 & 2.12 & 2.64 \\
\hline $\begin{array}{c}\text { Local Kn number at } \\
\text { nozzle exit based on } \mathrm{L}^{(* 2)}\end{array}$ & 0.024 & 0.024 & 0.029 \\
\hline $\begin{array}{c}\text { nozzle exit based on } \mathrm{L}^{(* 2)} \\
\text { nemmber at }\end{array}$ & 403 & 412 & 294 \\
\hline
\end{tabular}

${ }^{*} 1$ : LE : Leading edge angle[deg.]

$* 2: \mathrm{L}=0.05[\mathrm{~m}]$

(1) Uncertainty in measurement.

(2) Rotational temperature profiles at nozzle exit.

(3) Effects of three-dimensional flow.

(4) Rotational temperature profiles over plate for $T_{0}=670 \mathrm{~K}$.

First, the uncertainty in the measurement of the rotational temperature is estimated in the next section. Then the profiles of the rotational temperature at the nozzle exit are presented. Finally, the profiles of the rotational temperatures in a quasi-two-dimensional area are discussed after the effects of the three-dimensional flow over the flat plate are estimated. The coordinate system $(X, Y, Z)$ in the experiment is defined as follows. $X$ denotes the distance from the plate leading edge and is parallel to the plate surface. $Y$ denotes the distance from the plate surface in the direction normal to the plate. $Z$ is perpendicular to $X$ and $Y$ along the transverse direction of the plate. The origin is at the leading edge, on the symmetry line on the plate surface, and its radial location equals zero.

\subsection{Uncertainty in measurement}

The uncertainty in the measurement of the rotational temperature calculated from the fluorescence was esti-
Table 2 Measured average rotational temperature and difference of minimum and maximum values at each location

\begin{tabular}{|c|c|c|c|}
\hline $\begin{array}{c}\text { Location } \\
(X, Y, Z)[\mathrm{mm}]\end{array}$ & $\begin{array}{c}\text { Average } \\
\text { temperature[K] }\end{array}$ & $(\max .-\min ) / 2$. & $T_{0}[\mathrm{~K}]$ \\
\hline$(-5,0,0)$ & 154.85 & 6.7 & 670 \\
\hline$(-5,0,0)$ & 155.45 & 4.3 & 1000 \\
\hline$(10,2,0)$ & 292.48 & 13.4 & 670 \\
\hline$(10,2,0)$ & 279.90 & 6.5 & 1000 \\
\hline$(1,1,0)$ & 252.07 & 10.4 & 1000 \\
\hline
\end{tabular}

Table 3 Uncertainty in measurements of rotational temperature.

\begin{tabular}{|c|c|}
\hline Stagnation temperature $T_{0}[\mathrm{~K}]$ & Uncertainty $[\mathrm{K}]$ \\
\hline 670 & 13 \\
\hline 1000 & 10 \\
\hline
\end{tabular}

mated from about 50 measurements at three different locations near the leading edge of the plate. The location, experimental conditions and results are shown in Table 2. The slit width in the experiments at $T_{0}=1000 \mathrm{~K}$ is $120 \mathrm{~nm}$ and it is wider than that for $T_{0}=670 \mathrm{~K}(80 \mathrm{~nm})$ because the fluorescence at $T_{0}=1000 \mathrm{~K}$ was weaker than at $T_{0}=670 \mathrm{~K}$. The values of (max. rotational temperature min. rotational temperature)/2 in Table 2 are defined as the uncertainty in the measurement of rotational temperatures, and are listed in Table 3. The location uncertainty is estimated to $0.5 \mathrm{~mm}$ due to the half diameter of the electron beam.

\subsection{Rotational temperatures profiles at nozzle exit}

Figure 4 shows a comparison of the temperatures at the nozzle exit for $T_{0}=680 \mathrm{~K}$ (run 26). These are the rotational temperatures measured by EBFM, temperatures calculated from the measured Pitot pressures and temperature calculated by the surface static pressure at the nozzle exit (presented at $Y=0 \mathrm{~mm}$ ). The rotational temperatures obtained using the Muntz model and the RT model are plotted. Temperatures from the RT model are shown with error bars, whereas minimum and maximum values obtained from the Muntz model are plotted. The flat areas in both rotational temperatures from the RT model and temperatures obtained from the Pitot pressure have a width of about $30 \mathrm{~mm}$ near the nozzle center. The figure shows that the temperatures measured by the three different methods agree fairly well at the nozzle center. The difference between the maximum and the minimum rotational temperature distributions for the Muntz model is small near the 


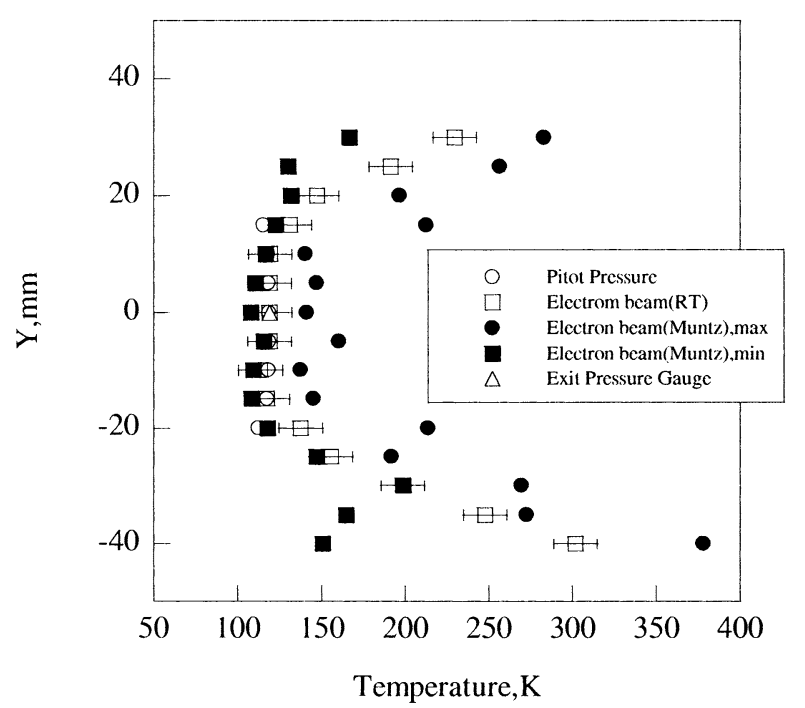

Fig. 4 Temperature profiles in nozzle exit plane for $p_{0}=965 \mathrm{~Pa}, T_{0}=680 \mathrm{~K}$

nozzle center; however, it is large near the nozzle walls due to nonequilibrium effects.

\subsection{Effects of three-dimensional flow}

In the flow over the flat plate, the deviation from an ideal flow situation originates from basically one effect. It is the interaction between the boundary layer developed in the nozzle and the sharp leading edge of the plate. Therefore, the region where this effect takes place was estimated in experimental run number 48 . The experiment has a stagnation temperature of about $1000 \mathrm{~K}$, which has the maximum Kn number.

Figure 5 shows the rotational temperature profiles downstream of the nozzle exit with the plate. Rotational temperatures are calculated using the RT model. The figure shows that in the range between $X=3$ and $15 \mathrm{~mm}$, the rotational temperature profiles at $Z=20 \mathrm{~mm}$ are $10 \sim 20 \mathrm{~K}$ larger than those at $Z=0,5,10 \mathrm{~mm}$; however, those in the region defined by $X=30 \mathrm{~mm}$ and $Y<5 \mathrm{~mm}$ are not dependent on the $\mathrm{Z}$ location.

From these results, the region with a planar flow over the plate is defined by $X<15 \sim 30 \mathrm{~mm}, Y<10 \sim 15 \mathrm{~mm}$ and $|Z|<10 \sim 20 \mathrm{~mm}$. The following investigation of nonequilibrium flow is mainly discussed in this region of planar flow.

\subsection{Rotational temperature profiles over plate for $T_{0}=670 \mathrm{~K}$}

Figure 6 shows the rotational temperature profiles at various $\mathrm{X}$ locations over the plate in run number 34 $\left(T_{0}=670 \mathrm{~K}\right)$. Rotational temperatures are calculated using the RT model. The rotational temperature near the plate increases for a location far from the leading edge. Rotational temperature distributions at $Y=1 \mathrm{~mm}$ are shown in Fig. 7. The data on the figure show that the relaxation length is about $15 \mathrm{~mm}$. In front of the leading edge $(X<0)$, the rotational temperature increases and is equal

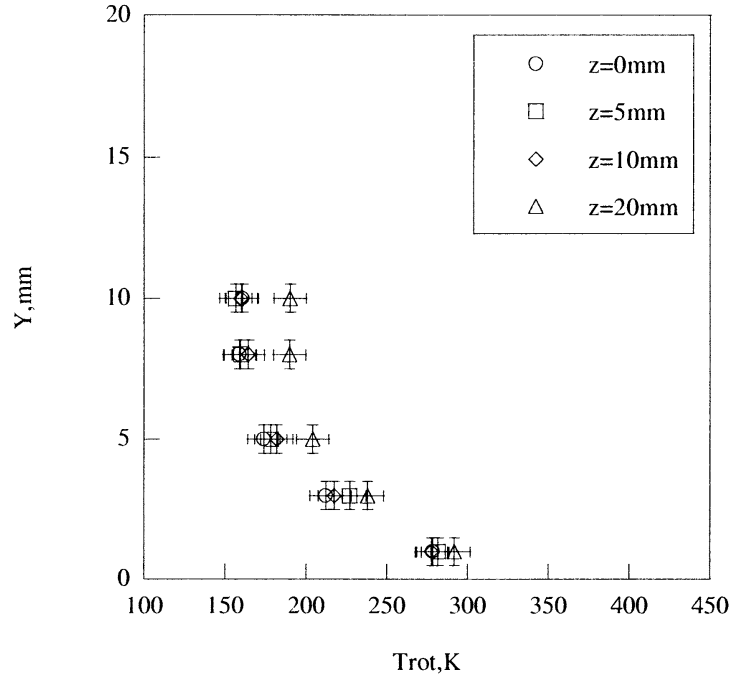

(a) $X=3 \mathrm{~mm}$

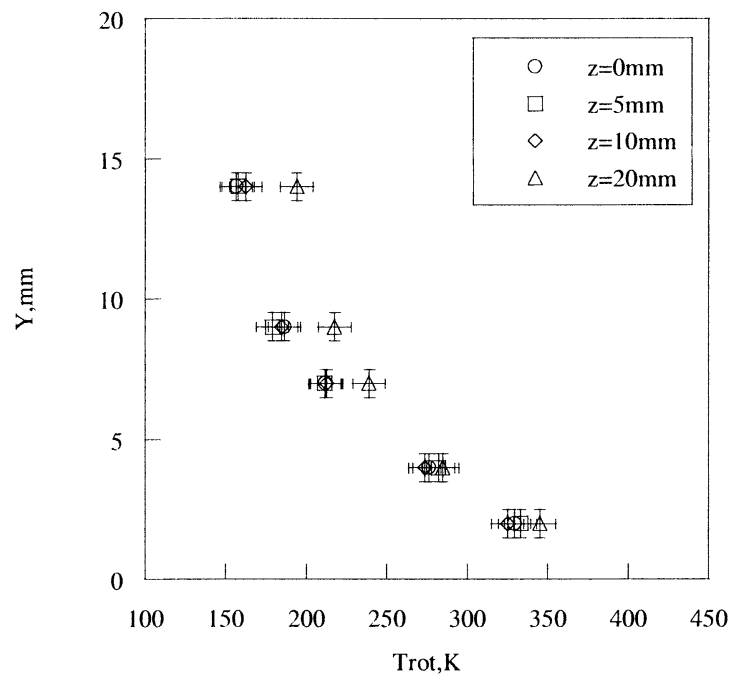

(b) $X=15 \mathrm{~mm}$

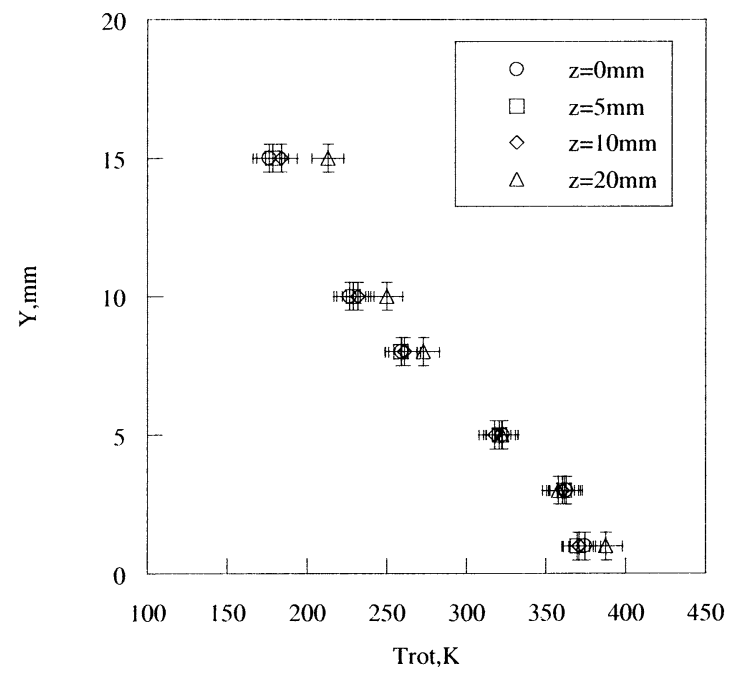

(c) $X=30 \mathrm{~mm}$

Fig. 5 Rotational temperature profiles with plate for $p_{0}=1271 \mathrm{~Pa}$ and $T_{0}=999 \mathrm{~K}$ 


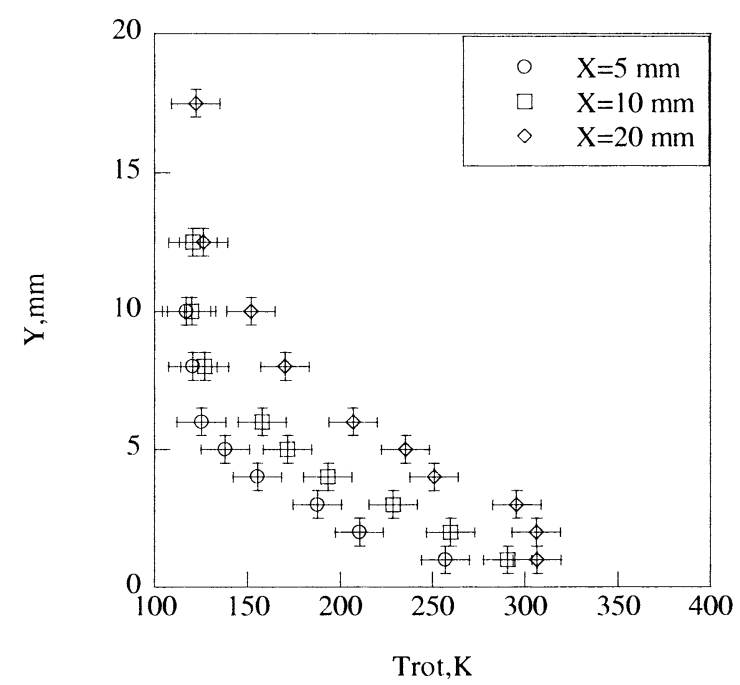

Fig. 6 Rotational temperature profiles over plate for $T_{0}=670 \mathrm{~K}$ $(Z=0 \mathrm{~mm})$

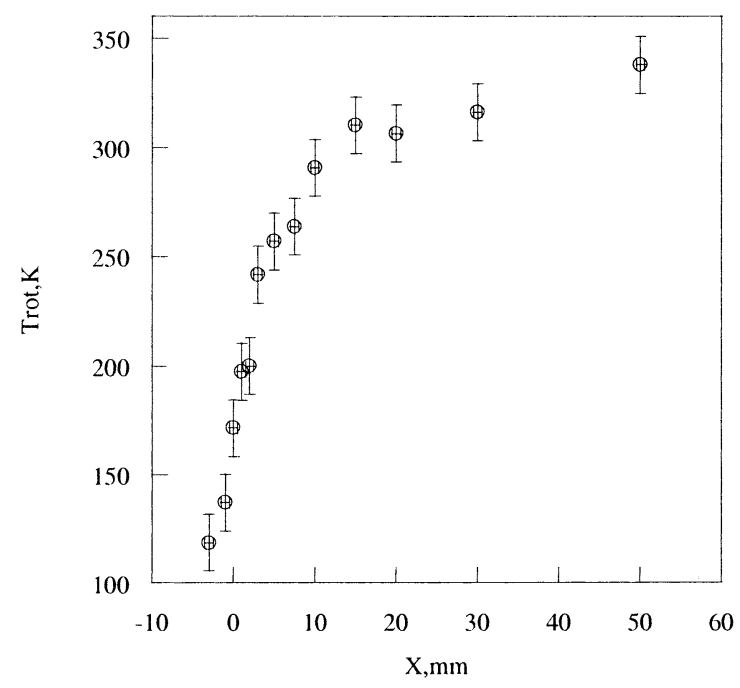

Fig. 7 Rotational temperature profiles for $T_{0}=670 \mathrm{~K}$ $(Y=1, Z=0 \mathrm{~mm})$

to $100 \sim 200 \mathrm{~K}$ at $X=0 \mathrm{~mm}$ due to the wedge side of the plate.

The rotational relaxation is discussed as follows. The analytical shock wave angle is $11.5 \mathrm{deg}$. for the freestream Mach number of 5 and the mean free path at the nozzle exit is $1.2 \mathrm{~mm}$ under this condition. Therefore, the mean free path along the plate surface is $6 \mathrm{~mm}$. The rotational collision number is approximately $2 \sim 4$ for the translational temperature of $100 \mathrm{~K}^{(20),(21)}$, then the rotational relaxation length is calculated to be $12 \sim 24 \mathrm{~mm}$. The rotational relaxation length in the present experimental results is approximately equal to the calculated value, even though the gas molecules collide with the plate surface in the actual conditions.

Figure 8 shows a comparison of rotational temperature distributions obtained using the RT and Muntz models. Rotational temperatures obtained by the Muntz model

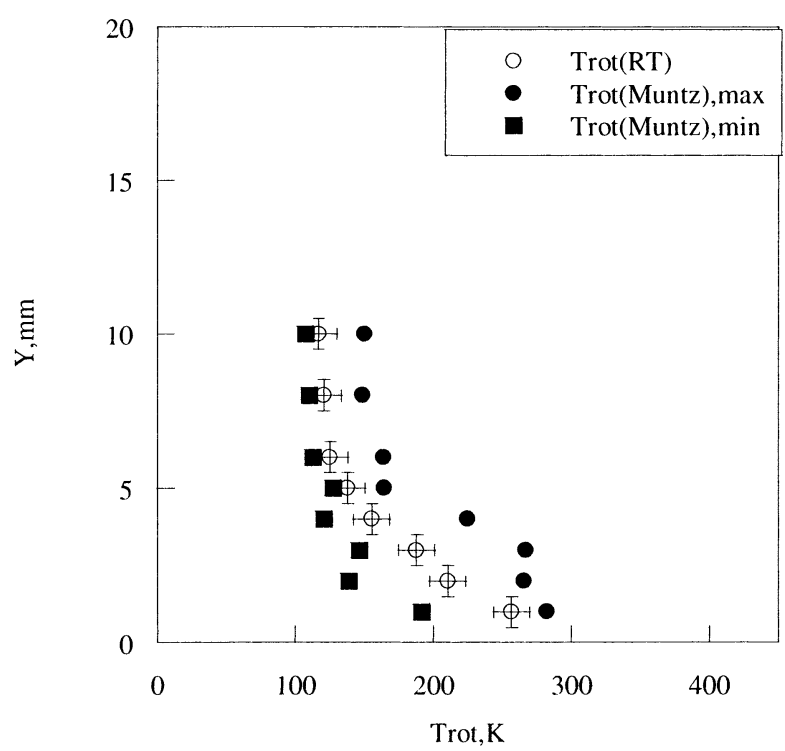

(a) $X=5 \mathrm{~mm}$

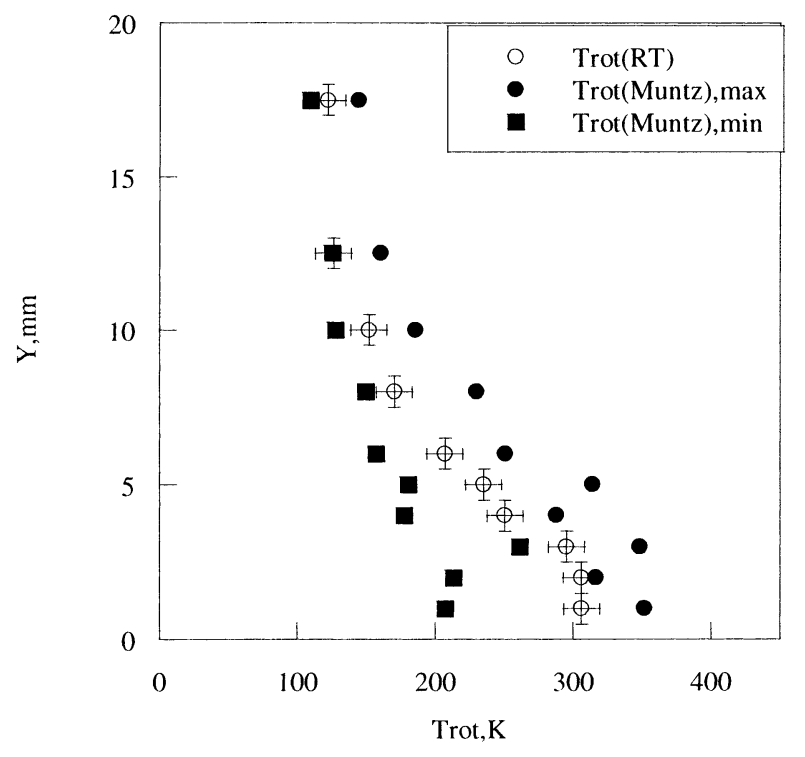

(b) $X=20 \mathrm{~mm}$

Fig. 8 Rotational temperature profiles for $T_{0}=670 \mathrm{~K}$ $(Z=0 \mathrm{~mm})$

exhibit large fluctuations, and the difference between maximum and minimum temperatures from this model becomes large in the vicinity of the plate rather than in the free stream. These results indicate that the nonequilibrium of the rotational temperatures exists near the flat plate surface.

Figure 9 shows $\log \left(I_{K} / P_{K}\right)$ vs. $E_{\text {rot }} / k$. MaxwellBoltzmann distributions are plotted in the figures as a reference. All the data exhibit a nonlinear variation, in agreement with the report of Robben and Talbot ${ }^{(18)}$, and they differ from the Maxwell-Boltzmann distributions. Therefore, the rotational energy distributions are shown to be in nonequilibrium. 


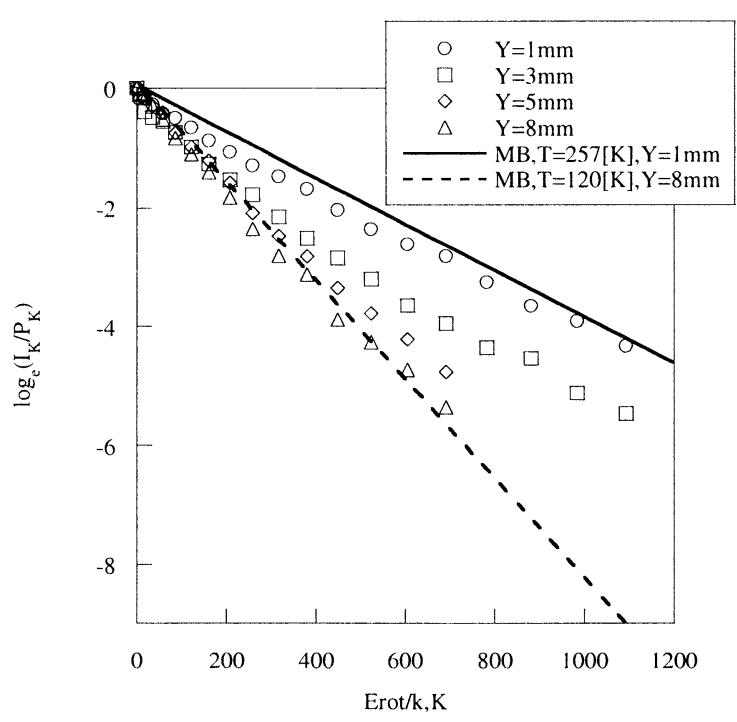

(a) $X=5 \mathrm{~mm}$

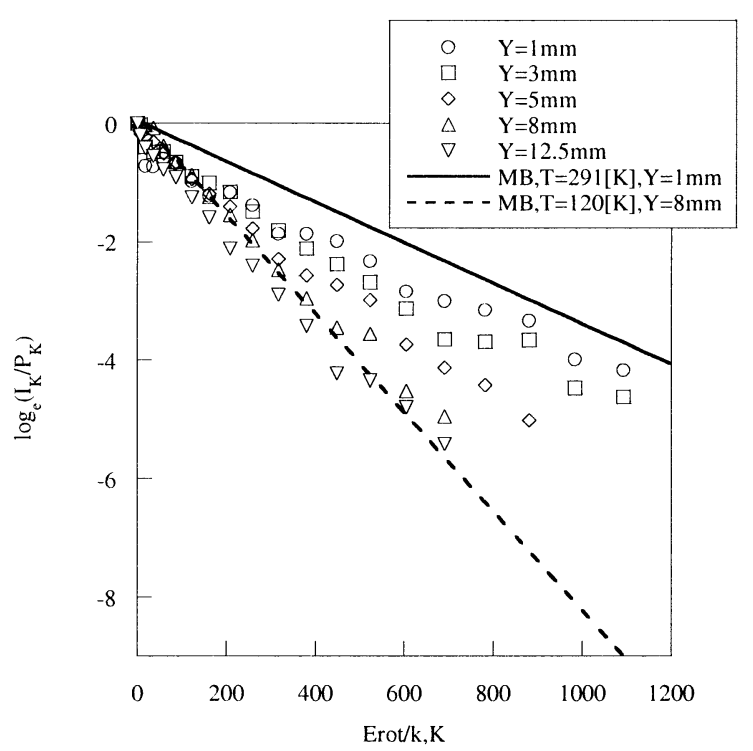

(b) $X=10 \mathrm{~mm}$

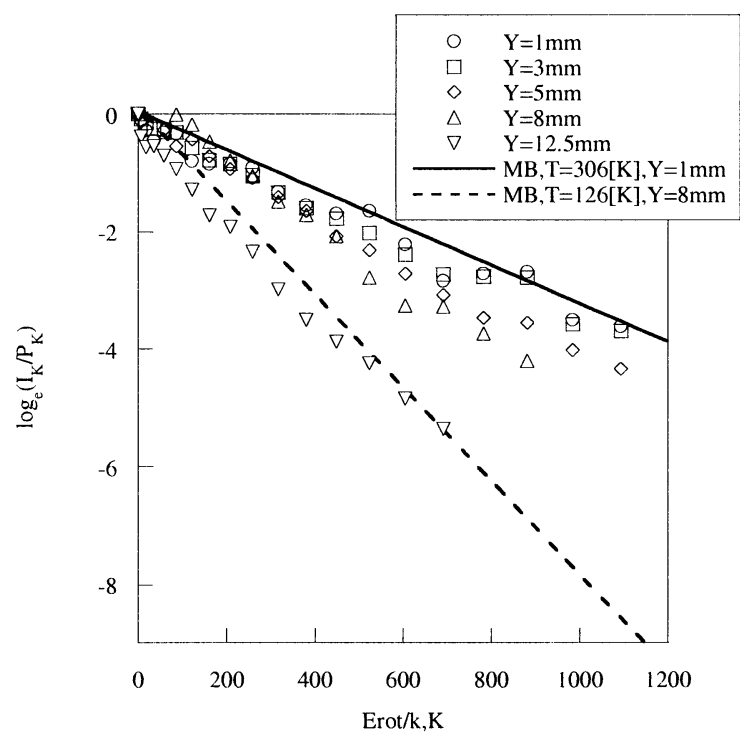

(c) $X=20 \mathrm{~mm}$

Fig. 9 Plots of $\log \left(I_{K} / P_{K}\right)$ vs. $E_{\text {rot }} / k$ for $T_{0}=670 \mathrm{~K}(Z=0 \mathrm{~mm})$

\section{Conclusions}

Experiments in a low-density wind tunnel were conducted to investigate rotational temperatures in shock wave and boundary layer interaction over a flat plate and the conclusions are as follows.

(1) The rotational temperatures near the leading edge of the plate were in nonequilibrium because the rotational energies differ from the Maxwell-Boltzmann distributions.

(2) The domain of the quasi two-dimensional flow over the plate, where the effects of the boundary layer developed in the nozzle were small, was limited to $X<$ 15 30 $\mathrm{mm}, Y<10 \sim 15 \mathrm{~mm}$ and $|Z|<10 \sim 20 \mathrm{~mm}$.

( 3 ) The rotational temperatures near the nozzle center were approximately equal to the temperature measured using the Pitot tube and the measured nozzle static pressure. Therefore, nonequilibrium near the nozzle center would be small.

(4) The rotational relaxation length near the leading edge corresponds to rotational collision numbers of $2 \sim 4$.

\section{Acknowledgements}

We would like to thank the late Prof. Alfred E. Beylich from RWTH Aachen for making available his low-density wind tunnel and the diagnostic facilities, as well as providing financial support for one of the authors (Nobuyuki Tsuboi) while staying at the laboratory in Aachen. Dr. Marc Havermann, now at French-German Research of Saint-Louis (ISL), Saint-Louis, designed and tested the heatable stagnation chamber; the never ending help and advice from Dr. Marc Havermann and Mr. Anshuman Awasthi during the experiments are greatly appreciated.

\section{References}

(1) Tsuboi, N., Yamaguchi, H. and Matsumoto, Y., Interaction between Shock Waves and Boundary Layer in Nonequilibrium Hypersonic Rarefied Flow (1st Report, Comparison with Continuum Model and LarsenBorgnakke Model), Trans. Jpn. Soc. Mech. Eng., (in Japanese), Vol.66, No.646, B (2000), pp.117-122.

( 2 ) Tsuboi, N., Yamaguchi, H. and Matsumoto, Y., Interaction between a Shock Wave and a Boundary Layer in a Nonequilibrium Hypersonic Rarefied Flow (2nd Report, Comparison of State for Translational and Rotational Energy Distributions), Trans. Jpn. Soc. Mech. Eng., (in Japanese), Vol.68, No.670, B (2002), pp.1653-1660.

( 3 ) Tsuboi, N. and Matsumoto, Y., Interaction between a Shock Wave and a Boundary Layer in a Nonequilibrium Hypersonic Rarefied Flow (3rd Report, Effects of Gas-Surface Interaction Model), Trans. Jpn. Soc. Mech. Eng., (in Japanese), Vol.68, No.670, B (2002), pp.1661-1668.

( 4 ) Tokumasu, T. and Matsumoto, Y., Dynamic Molecular 
Collision (DMC) Model for Rarefied Gas Flow Simulations by the DSMC Method, Phys. Fluids, Vol.11, No.7 (1999), pp.1907-1920.

( 5 ) Yamanishi, N. and Matsumoto, Y., Multistage GasSurface Interaction Model for the Direct Simulation Monte Carlo Method, Phys. Fluids, Vol.11, No.11 (1999), pp.3540-3551.

( 6 ) Bird, G.A., Molecular Gas Dynamics, (1976), Clarendon Press, Oxford.

(7) Nanbu, K., Stochastic Solution Method of the Model Kinetic Equation for Diatomic Gas, J. Phys. Soc. Jpn., Vol.49 (1990), pp.2042-2049.

( 8 ) Muntz, E.P., Static Temperature Measurements in a Flowing Gas, Phys. Fluids, Vol.5, No.1 (1962), pp.8090.

(9) Robben, F. and Talbot, L., Measurement of Shock Wave Thickness by the Electron Beam Fluorescence Method, Phys. Fluids, Vol.9, No.4 (1966), pp.633-643.

(10) Tsuboi, N. and Matsumoto, Y., Interaction between a Shock Wave and a Boundary Layer in a Nonequilibrium Hypersonic Rarefied Flow (4th Report, Nozzle Design for Low Density Wind Tunnel), Trans. Jpn. Soc. Mech. Eng., (in Japanese), Vol.71, No.706, B (2005), pp.1579-1587.

(11) Tsuboi, N. and Matsumoto, Y., Experimental and Numerical Study of Hypersonic Rarefied Gas Flow over Flat Plates, AIAA Journal, Vol.43, No.6 (2005), pp.1243-1255.

(12) Beylich, A.E. and Richarz, H.P., Expansion of Gas Mixtures in Free Jet, Proc. 14th Int. Symp. Rarefied Gas Dyn., (1984), pp.457-466.

(13) Tsuboi, N., Structure of Non-Equilibrium Interaction between Shock Waves and Boundary Layer in Hypersonic Rarefied Flow, Ph.D. Thesis in Department of Mechanical Engineering, The University of Tokyo, (2000).

(14) Muntz, E.P., The Electron Beam Fluorescence Technique, AGARD 132, (1968).

(15) Marrone, P.V., Temperature and Density Measurement in Free Jets and Shock Waves, Phys. Fluids, Vol.10, No.3 (1967), pp.521-538.

(16) Marrone, P.V., Rotational Temperature and Density Measurements in Underexpanded Jets and Shock Waves Using an Electron Beam Fluorescence, UTLAS Report, Vol.112 (1966).

(17) Robben, F. and Talbot, L., Measurement of Rotational Temperatures in a Low Density Wind Tunnel, Phys. Fluids, Vol.9, No.4 (1966), pp.644-652.

(18) Robben, F. and Talbot, L., Experimental Study of the Rotational Distribution Function of Nitrogen in a Shock Wave, Phys. Fluids, Vol.9, No.4 (1966), pp.653662.

(19) Tokumasu, T. and Matsumoto, Y., An Improved Method for the Estimation of Rotational Temperature with a Weak Electron Beam, Trans. Jpn. Soc. Mech. Eng., (in Japanese), Vol.68, No.676, B (2002), pp.3352-3359.

(20) Parker, J.G., Rotational and Vibrational Relaxation in Diatomic Gases, Phys. Fluids, Vol.2, No.4 (1959), pp.449-462.

(21) Lumpkin III, F.E. and Park, C., A New Rotational Relaxation Model for Use in Hypersonic Computational Fluid Dynamics, AIAA Paper 89-1737, (1989). 